\title{
A narrative review of low-dose rivaroxaban in patients with atherothrombotic cardiovascular disease: vascular protection beyond anticoagulation
}

\author{
Bruno Miguel Lopes Rocha, Gonçalo José Lopes da Cunha, Carlos Manuel Tavares Aguiar \\ Cardiology Department, Hospital de Santa Cruz, Centro Hospitalar Lisboa Ocidental, Lisbon, Portugal \\ Contributions: (I) Conception and design: BML Rocha, CMT Aguiar; (II) Administrative support: All authors; (III) Provision of study materials or \\ patients: BML Rocha, CMT Aguiar; (IV) Collection and assembly of data: BML Rocha, GJL da Cunha; (V) Data analysis and interpretation: All \\ authors; (VI) Manuscript writing: All authors; (VII) Final approval of manuscript: All authors. \\ Correspondence to: Bruno Miguel Lopes Rocha. Cardiology Department, Hospital de Santa Cruz, Centro Hospitalar Lisboa Ocidental, Av. Prof. Dr. \\ Reinaldo dos Santos, 2790-134 Carnaxide, Lisbon, Portugal. Email: bruno.rocha@campus.ul.pt.
}

\begin{abstract}
Major cardiovascular (CV) events often complicate the natural history of apparently stable atherothrombotic cardiovascular disease (CVD) despite appropriate guideline-based preventive treatment. This finding has been termed residual risk and it has been the focus of recent investigation. New and revisited targets to tackle this so-called residual risk have been proposed, including antithrombotic treatment intensification, further lowering targets of low-density lipoprotein (LDL) cholesterol, novel oral antidiabetic agents with a $\mathrm{CV}$ benefit, and drugs to reduce systemic inflammation. In this narrative review, we discuss the evidence, mechanisms and gaps in knowledge concerning the vascular protection derived from lowdose (2.5 $\mathrm{mg}$ twice daily) rivaroxaban. On this topic, the main trials (ATLAS ACS 2-TIMI 51, COMPASS and VOYAGER PAD), will be summarized in a comprehensive manner. Indeed, these have shown that a drug developed to prevent thrombus formation (selective Factor Xa inhibition) reduced events that were traditionally platelet-related in concept. Moreover, we propose a simple evidence-based clinically oriented algorithm to thoroughly identify patients at increased risk and who may benefit from this strategy in different clinical scenarios. Low-dose rivaroxaban portrays a novel promising era in atherothrombotic CVD prevention, providing a mechanistic protection beyond traditional strategies in patients overwhelmed by recurrent dismal events.
\end{abstract}

Keywords: Atherothrombotic cardiovascular disease (atherothrombotic CVD); rivaroxaban; direct oral anticoagulants (DOACs); coronary artery disease (CAD); peripheral artery disease (PAD)

Submitted Oct 17, 2020. Accepted for publication Dec 28, 2020.

doi: $10.21037 / \mathrm{cdt}-20-859$

View this article at: http://dx.doi.org/10.21037/cdt-20-859

\section{Introduction: beyond traditional secondary prevention in atherothrombotic cardiovascular disease (CVD)}

Atherothrombotic CVD encompasses cerebrovascular disease, coronary artery disease (CAD) and peripheral artery disease (PAD). Despite outstanding available effective guideline-based preventive therapies, these patients are still at risk of significant, and often clinically meaningful, ischaemic events. This so-called residual risk is particularly expressive in those with symptomatic atherothrombotic CVD (1-4). The REduction of Atherothrombosis for Continued Health (REACH) registry assessed major cardiovascular $(\mathrm{CV})$ outcomes in patients $(n=64,977)$ with chronic atherothrombotic CVD or three or more $\mathrm{CV}$ atherosclerosis risk factors. In the secondary prevention subgroup $(n=53,390)$, despite recommended medical therapy (including lipid-lowering agents and antithrombotic drugs, and antihypertensive and anti-diabetic drugs, 

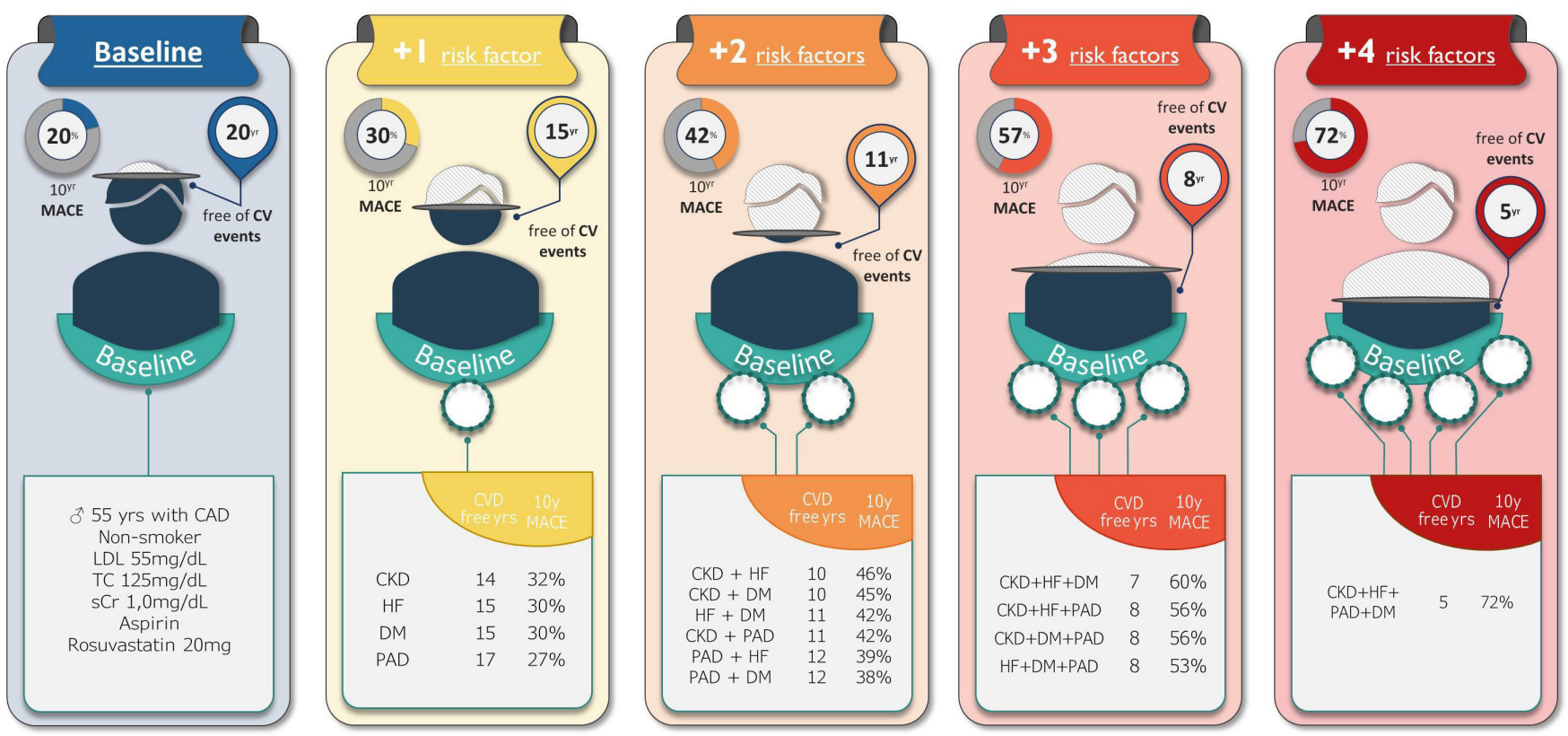

Figure 1 Ten-year risk of major adverse CV events estimated by the SMART-REACH model for a 55-year-old male non-smoker patient with CAD receiving optimal medical treatment for arterial hypertension (SBP $130 \mathrm{mmHg}$ ), added to low-dose aspirin and rosuvastatin $20 \mathrm{mg}$ per day (total cholesterol $125 \mathrm{mg} / \mathrm{dL}$ and LDL-cholesterol $55 \mathrm{mg} / \mathrm{dL}$ ), and normal renal function (sCr of $1.0 \mathrm{mg} / \mathrm{dL}$ ). Under these conditions, the MACE risk is $20 \%$ and the expected CVD-free survival is 20 years, but the latter will be reduced to 17 years if PAD is present, 15 years if either DM or HF is present, 14 years if CKD is present, 12 years if both DM and PAD are present, and only 8 years if both DM and PAD are present together with either CKD or HF. CV, cardiovascular; SBP, systolic blood pressure; LDL, low-density lipoprotein; MACE, major adverse cardiovascular events; CVD, cardiovascular disease; PAD, peripheral artery disease; DM, diabetes mellitus; HF, heart failure; CKD, chronic kidney disease (assuming sCr of $2.0 \mathrm{mg} / \mathrm{dL}$ ); sCr, serum creatinine.

whenever applicable), the observed annual rate of $\mathrm{CV}$ death, myocardial infarction (MI) or stroke was $4.6 \%$. This rate further increased to $14.4 \%$ when considering the composite of $\mathrm{CV}$ death, MI, stroke or hospitalization for unstable angina, transient ischaemic attack (TIA) or worsening PAD $(1,2)$. These findings demonstrate that adverse vascular events are frequent complications in the natural history of apparently stable atherothrombosis on appropriate treatment, namely antiplatelet and lipid-lowering agents, thus corroborating the state-of-the-art concept of chronic coronary syndromes (CCSs) and dethroning the outdated stable designation.

Interestingly, the REACH registry additionally found a higher rate of $\mathrm{CV}$ death, MI or stroke in secondary prevention patients with a prior ischaemic event more than 1 year before enrolment compared to those without history of acute ischaemic events at inclusion $(1,2)$. Currently, dual antiplatelet therapy (DAPT) is recommended up to 1 year after an acute coronary syndrome (ACS), yet the aforementioned findings challenge the de-escalation approach and call for additional (or alternative) strategies to mitigate deceptive stability.

The risk of a first or recurrent ischaemic event amongst patients on secondary prevention treatment is highly variable (5). An ideal management strategy includes risk stratification to select those patients who could benefit the most from new drugs and/or treatment intensification. The Secondary Manifestations of Arterial Disease (SMART)REACH model was recently developed to predict life expectancy without recurrent $\mathrm{CV}$ events in a population with established CVD (6). This mathematical model collects data on the following variables: diabetes mellitus $(\mathrm{DM})$, heart failure (HF), PAD, atrial fibrillation, current smoking, chronic kidney disease (CKD), blood pressure (BP) and total cholesterol. An illustration of how much these variables influence prognosis is presented in Figure 1. By using the example of a 55-year-old male non-smoker patient with $\mathrm{CAD}$, treated with rosuvastatin ( $20 \mathrm{mg}$ per day) 
Table 1 Trials focused on strategies to reduce major CV events in patients at high risk of atherothrombosis

\begin{tabular}{|c|c|c|c|c|c|}
\hline Characteristics & PEGASUS (7) & FOURIER (8) & CANTOS (9) & EMPA-REG (10) & COMPASS (11) \\
\hline Population & $\mathrm{Ml}>1-3$ ys & $\mathrm{CVD}+\mathrm{LDL} \geq 70 \mathrm{mg} / \mathrm{dL}$ & $\mathrm{Ml}+\mathrm{hs}-\mathrm{CRP} \geq 2 \mathrm{mg} / \mathrm{L}$ & CVD + type 2 DM & CAD and/or PAD \\
\hline All-cause death & - & - & - & $\downarrow 32 \%, N^{N} T_{1-y r}=385$ & $\downarrow 18 \%, \mathrm{NNT}_{23-\mathrm{mo}}=143$ \\
\hline CV death & - & - & - & $\downarrow 38 \%, \mathrm{NNT}_{1-\mathrm{yr}}=455$ & $\downarrow 22 \%, \mathrm{NNT}_{23-\mathrm{mo}}=200$ \\
\hline MI & $\downarrow 16 \%, \mathrm{NNT}_{3-\mathrm{yr}}=139$ & $\downarrow 27 \%, \mathrm{NNT}_{26-\mathrm{mo}}=83$ & $\downarrow 16 \%, \mathrm{NNT}_{1-\mathrm{yr}}=270$ & - & - \\
\hline Stroke & $\downarrow 25 \%, N^{N N T_{3-y r}}=213$ & $\downarrow 21 \%, \mathrm{NNT}_{26-\mathrm{mo}}=250$ & - & - & $\downarrow 42 \%, \mathrm{NNT}_{23-\mathrm{mo}}=143$ \\
\hline Adverse events & Non-fatal bleeding, dyspnea & Injection site reaction & Fatal infection & Genital infection & Non-fatal bleeding \\
\hline
\end{tabular}

Dashed cells (-) correspond to outcomes that did not meet statistical significance. CV, cardiovascular; MI, myocardial infarction; CVD, cardiovascular disease; LDL, low-density lipoprotein; hs-CRP, high sensitivity C-reactive protein; DM, diabetes mellitus; CAD, coronary artery disease; PAD, peripheral artery disease; NNT, number needed to treat to prevent an event per unit of time.

and low-dose aspirin, presenting on-target BP and total cholesterol, and normal renal function, it shows that CVDfree survival can vary up to 4-fold based on the presence of additional risk factors collected by the SMART-REACH model.

New (or revisited) targets have been proposed to extend the therapeutic armamentarium and improve prognosis in well-selected patients with established CVD and at higher risk of first or recurrent ischaemic events (7-14). Fortunately, several recently conducted breakthrough clinical trials focusing on different strategies have shown positive results (Table 1):

(I) Antithrombotic therapy intensification: e.g., prolonged DAPT (ticagrelor plus aspirin) (7) or adding direct Factor $\mathrm{Xa}$ inhibition (rivaroxaban) to aspirin (11,14);

(II) Intensified low-density lipoprotein (LDL) cholesterol reduction: e.g., ezetimibe (15) and proprotein convertase subtilisin/kexin type 9 (PSCK9) inhibitors (8);

(III) Antidiabetic drugs with a CV benefit: e.g., sodiumglucose transport protein 2 (SLGT-2) inhibitors (10) and glucagon-like peptide-1 agonists (13);

(IV) Drugs targeting systemic inflammation: e.g., canakinumab [interleukin (IL)-1 antagonist] (9) and low-dose colchicine (12).

The management of the residual risk of ischaemic vascular events remains controversial, although most strategies comprise of targeting pathways strongly implicated in disease progression. Hypothetically, targeting multiple mechanisms at the same time may associate with even better outcome. Furthermore, clinical features that facilitate the selection of patients in whom these interventions have demonstrated a (cost-effective) net clinical benefit have been identified (16). However, there are several unknowns, calling for urgent action in a matter of utmost importance.

The aim of this review is to discuss the potential mechanisms and added benefits (of the so-called vascular protection) derived from low-dose rivaroxaban. In this review, the term 'vascular protection' describes a comprehensive therapeutic strategy that prevents arterial ischemic events, such as MI and ischemic stroke. Studies published in English on this matter were searched in MEDLINE and relevant articles, as considered by the authors, are discussed within this review. We present the paper in accordance with the Narrative Review reporting checklist (available at http://dx.doi.org/10.21037/cdt-20859).

\section{Oral anticoagulation provides vascular protection beyond antithrombotic activity: evidence from clinical trials}

Platelet activation and aggregation have traditionally been perceived to play a dominant role in the pathogenesis of 
atherosclerosis and atherothrombosis, but recent evidence has accumulated on the contribution of coagulation proteins to these processes (4). Two landmark trials in postMI patients-WARIS (17) and WARIS II (18)-pioneered the concept of oral anticoagulation in CAD. These demonstrated that warfarin taken alone or in combination with aspirin was superior in reducing the risk of recurrent MI (compared to placebo or to single antiplatelet therapy with aspirin, respectively). Indeed, these findings corroborate that anticoagulation can prevent arterial events. However, it should be noted that these trials were performed in the pre-statin era and largely before extensive use of percutaneous coronary intervention. Furthermore, a benefit with anticoagulation in post-MI patients has been suggested in the phase II ESTEEM (19) and REDEEM (20) trials, with ximelagatran and dabigatran, albeit hepatotoxicity and/or bleeding limited their use, respectively. Similarly, the APPRAISE-2 trial (21) with apixaban was stopped early due to an increase in bleeding events, having had a potential benefit in the subgroup of patients without prior stroke (22).

In the current era of direct oral anticoagulants (DOACs), several studies in patients with atherothrombotic CVD have clearly demonstrated the benefit of adding a low dose of rivaroxaban (2.5 $\mathrm{mg}$ twice daily, also known as vascular dose) to standard treatment for superior prevention of recurrent vascular events. Of these, the ATLAS ACS 2-TIMI 51 (23) COMPASS (11) and VOYAGER PAD (24) trials will be discussed. This dosing regimen was chosen on the basis of the ATLAS ACS-TIMI 46 results (25), which showed a graded increase in bleeding across total daily doses of $5 \mathrm{mg}$ up to $20 \mathrm{mg}$, while efficacy was noted at the lowest dose.

\section{The ATLAS ACS 2-TIMI 51 trial}

The Anti-Xa Therapy to Lower Cardiovascular Events in Addition to Standard Therapy in Subjects with Acute Coronary Syndrome-Thrombolysis in Myocardial Infarction 51 (ATLAS ACS 2-TIMI 51) trial was a doubleblind multicentre study enrolling stable ACS patients. To be eligible, those under 55 years of age had to have either DM or a previous MI. Patients were randomized to twice-daily 2.5 or $5 \mathrm{mg}$ rivaroxaban or placebo. The primary efficacy endpoint was a composite of $\mathrm{CV}$ death, $\mathrm{MI}$ or stroke. The primary safety endpoint was TIMI major bleeding not related to coronary artery bypass grafting (CABG) (23). Overall, 15,526 patients were enrolled (mean age 62 years; $75 \%$ male, often with $\mathrm{CV}$ risk factors). Patients were treated according to guidelines with DAPT (93\%), statins (84\%) and beta-blockers $(66 \%)$ plus myocardial revascularization $(60 \%)$.

At a mean follow-up of 13 months, the addition of rivaroxaban $2.5 \mathrm{mg}$ twice daily (vascular dose) to standard therapy compared to placebo significantly reduced the primary efficacy endpoint [relative risk reduction (RRR): $16 \% ; \mathrm{P}=0.02$ ]. These results were primarily driven by a reduction of $\mathrm{CV}$ death (RRR: $34 \% ; \mathrm{P}=0.002$ ). Moreover, this strategy also reduced all-cause death (RRR: $32 \%$; $\mathrm{P}=0.004)$, and the benefits were sustained after stopping thienopyridines (at maximum follow-up of 31 months) (23). Interestingly, rivaroxaban vascular dose reduced stent thrombosis events [probable or definite, as per Academic Research Consortium (ARC) definition] compared to placebo ( $2.9 \%$ vs. $4.5 \%$; RRR: $39 \%$; $\mathrm{P}=0.002)$. The latter result is truly remarkable as stent thrombosis is, at least in concept, a platelet-dependent pathophysiological phenomenon. Indeed, this hypothesis had been demonstrated in the Stent Anticoagulation Restenosis Study (SARS) study, in which warfarin was inferior to ticlopidine in preventing stent thrombosis events in a population who received non-drug eluting stents and aspirin (26).

As for safety outcomes, even though rivaroxaban vascular dose significantly increased the risk of major bleeding, roughly tripling the risk of TIMI major bleeding not related to $\mathrm{CABG}$ and doubling intracranial haemorrhage, there were no significant differences in fatal bleeding compared to placebo in the ATLAS ACS 2-TIMI 51 trial.

\section{The COMPASS trial}

The Cardiovascular Outcomes for People Using Anticoagulation Strategies (COMPASS) trial was a doubleblind multicentre study that enrolled patients with stable atherosclerotic $\mathrm{PAD}, \mathrm{CAD}$ or both. $\mathrm{CAD}$ patients younger than 65 years required atherosclerosis in two vascular beds or two CV risk factors. Key exclusion criteria included a formal indication for DAPT and HF with left ventricular ejection fraction (LVEF) $<30 \%$ or New York Heart Association (NYHA) III-IV. Patients were randomized to aspirin $100 \mathrm{mg}$, rivaroxaban $5 \mathrm{mg}$ bid, or rivaroxaban $2.5 \mathrm{mg}$ bid plus aspirin $100 \mathrm{mg}$. The primary efficacy endpoint was a composite of $\mathrm{CV}$ death, MI or stroke. The primary safety endpoint included fatal bleeding, symptomatic bleeding into a critical organ, bleeding into a surgical site requiring reoperation, and bleeding leading to hospitalization or acute care (11). Overall, 27,395 patients were enrolled (mean 
Table 2 Net clinical benefit analysis of high-risk subgroups enrolled in COMPASS

\begin{tabular}{|c|c|c|c|}
\hline Characteristics & \multicolumn{2}{|c|}{ Incidence of net clinical benefit* at 24 months } & $\frac{\text { Prevented events per } 1,000 \text { pts }}{\text { DPI vs. aspirin }}$ \\
\hline Overall population & 5.0 & 6.6 & 15.8 \\
\hline \multicolumn{4}{|l|}{ Special populations } \\
\hline Polyvascular disease & 7.6 & 11.1 & 35.2 \\
\hline eGFR $<60 \mathrm{~mL} / \mathrm{min}$ & 7.6 & 9.8 & 22.3 \\
\hline DM & 6.5 & 8.5 & 20.3 \\
\hline
\end{tabular}

There was no significant interaction between treatment regimen (DPI vs. aspirin) and studied subgroups regarding efficacy, safety or net clinical benefit. *, Net clinical benefit was defined as a composite of CV death, MI, stroke, fatal bleeding or symptomatic bleeding in critical organ. DPI, dual pathway inhibition (vascular dose rivaroxaban plus aspirin); HF, heart failure; eGFR, estimated glomerular filtration rate; DM, diabetes mellitus.

age 68 years; $78 \%$ male, $91 \%$ with $\mathrm{CAD}$ and 27\% $\mathrm{PAD}$ ). Patients were often on lipid-lowering agents (90\%) and had well controlled CV risk factors.

At a mean follow-up of 23 months (after early termination, as per trial protocol due to achievement of interim efficacy threshold), rivaroxaban $2.5 \mathrm{mg}$ twice daily with aspirin [hereafter referred as dual pathway inhibition (DPI), unless otherwise specified] was associated with a significant RRR of $24 \%$ [ $1.3 \%$ absolute risk reduction (ARR)] of the primary endpoint compared to aspirin. The findings were mainly driven by reductions in each component of the primary endpoint: $\mathrm{CV}$ death (RRR: $22 \% ; \mathrm{P}=0.02$ ), $\mathrm{MI}$ (RRR: $14 \% ; \mathrm{NS}$ ) and stroke (RRR: $42 \% ; \mathrm{P}<0.001)$. Moreover, there was a marginal significant reduction in all-cause death (RRR: $18 \% ; \mathrm{P}=0.01$ ). As for the primary safety endpoint, although DPI compared to aspirin alone was significantly associated with a higher risk of major haemorrhagic events $(3.1 \%$ vs. $1.9 \% ; \mathrm{P}<0.001)$, mostly due to an increase in nonfatal gastrointestinal bleeding $(1.5 \%$ vs. $0.7 \% ; \mathrm{P}<0.001)$, the rate of fatal bleeding or intracranial haemorrhage was low and no different between groups. A prespecified net clinical benefit outcome that accounted for both efficacy and safety (i.e., CV death, MI, stroke, fatal haemorrhage or symptomatic bleeding into a critical organ) also favoured DPI, with a $1.2 \%$ ARR in events $(\mathrm{P}<0.001)$. This net clinical benefit (and potential cost-effectiveness) was particularly significant in patients at higher risk of ischaemic events (polivascular arterial disease, HF with LVEF $>30 \%$ and NYHA I-II symptoms, CKD and DM)
(Table 2) (11).

The COMPASS trial results contribute significantly to reducing morbidity and mortality in patients with PAD. The rationale for antithrombotic treatment intensification in PAD had been previously tested, yet none of the interventions put on trial had proven benefit or a reasonable net clinical benefit. In the Examining Use of Ticagrelor in Peripheral Artery Disease (EUCLID) trial, enrolling patients with symptomatic $\mathrm{PAD}$, ticagrelor was not superior to clopidogrel in reducing the composite primary endpoint of $\mathrm{CV}$ death, $\mathrm{MI}$ or ischaemic stroke, nor admission due to acute limb ischaemic or lower-limb revascularization (27). In the subgroup of PAD patients enrolled in the Thrombin Receptor Antagonist in Secondary Prevention of Atherothrombotic Ischemic Events (TRA 2P-TIMI 50) trial, voraxapar ( $2.5 \mathrm{mg}$ daily) was superior to standard therapy regarding the risk of hospitalization for acute limb ischaemic and the rate of lower-limb revascularizations, but no benefit was observed in the composite primary endpoint of CV death, MI or ischaemic stroke (28). Contrasting with the former, in the subgroup of PAD patients enrolled in the COMPASS trial, DPI compared to aspirin reduced the primary composite endpoint of $\mathrm{CV}$ death, $\mathrm{MI}$ or ischaemic stroke (RRR: 28\%), as well as acute limb ischaemic events (RRR: 44\%), total vascular amputations (RRR: 60\%) and major amputations (RRR: 70\%) with a favourable net clinical benefit. Indeed, a composite of CV death, MI, stroke, fatal haemorrhage or symptomatic bleeding into a critical organ favoured DPI compared to aspirin alone in 
patients with either stable PAD or carotid artery disease (RRR: 25\%; P=0.011) $(11,29)$.

\section{The VOYAGER PAD trial}

Recently, the results of the Vascular Outcomes Study of ASA Along with Rivaroxaban in Endovascular or Surgical Limb Revascularization for PAD (VOYAGER PAD) trial were published (24). This was a double-blind multicentre study that enrolled patients aged 50 years or older with moderate to severe symptomatic PAD and a successful peripheral revascularization. Key exclusion criteria included planned long-term DAPT. Patients were randomized to rivaroxaban $2.5 \mathrm{mg}$ bid added to aspirin $v s$. aspirin alone. The primary efficacy outcome was a composite of acute limb ischaemic, major amputation for vascular causes, MI, ischaemic stroke or $\mathrm{CV}$ death. Overall, 6,564 patients were enrolled (median age 67 years; 74\%, with a high burden of $\mathrm{CV}$ risk factors). Patients were often on statins $(80 \%)$ and clopidogrel $(50 \%)$ at baseline. Most (65\%) were treated with an endovascular procedure, while the remainder were surgically treated.

At a median 28 months follow-up, adding vascular dose rivaroxaban to aspirin significantly reduced the primary efficacy endpoint (RRR: $15 \%$; $\mathrm{P}=0.009$ ). The results were primarily driven by a reduction in acute limb ischaemic events $(\mathrm{P}=0.03)$, with an early separation of the KaplanMeier curves and consistent results amongst subgroups. However, there was no significant reduction in all-cause mortality $(\mathrm{P}=0.34)$ with this strategy. As for safety outcomes, DPI was associated with increased International Society on Thrombosis and Haemostasis (ISTH) major bleeding compared to aspirin monotherapy, but no differences in intracranial haemorrhage or fatal bleeding were noted.

\section{Summary of evidence from clinical trials}

In summary, in patients with $\mathrm{CAD}$ or symptomatic $\mathrm{PAD}$ at higher ischaemic risk, it is appropriate to consider adding the so-called vascular dose of rivaroxaban $(2.5 \mathrm{mg}$ twice daily) to standard aspirin in order to reduce the risk of CV death, MI, stroke, acute limb ischaemia and major amputation. Given the increased risk for major bleeding events (excluding fatal and symptomatic haemorrhage into a critical organ), this strategy should be considered on a case-by-case basis in patients whose thrombotic risk might outweigh the bleeding risk. However, adequate benefit-risk tools to identify and select such patients are expeditiously needed. In Figure 2 based on the inclusion and exclusion criteria of the aforementioned discussed trials, we propose an evidence-based and clinically oriented algorithmic approach to identify those who may benefit the most from introducing low-dose rivaroxaban. This is a promising strategy in patients overwhelmed by recurrent dismal events and at relatively low risk of haemorrhagic events.

\section{Oral anticoagulation provides vascular protection beyond antithrombotic activity: mechanisms with a focus on Factor Xa activity}

Remarkably, the ATLAS ACS 2-TIMI 51 (23), COMPASS (11) and VOYAGER PAD (24) trials have shown that rivaroxaban, a drug developed to prevent thrombus formation by selectively inhibiting Factor Xa, reduced atherothrombotic events that were traditionally thought to be primarily platelet-related (31). Here we discuss three main foundations to appreciate this evidence:

(I) Anticoagulants ultimately reduce thrombin generation or inhibit the effects of thrombin, but this coagulation protein is also the most potent endogenous platelet activator which exerts this effect by binding to protease-activated receptors (PARs) on the platelet surface; hence, there is a significant interplay between the coagulation cascade and platelets;

(II) Factor $\mathrm{Xa}$ and thrombin are serine proteases and both activate PARs; yet, these receptors are found not only on platelets but also on several cells implicated in inflammation and atherogenesis, such as endothelial cells and macrophages, to name a few; hence, an anticoagulant such as rivaroxaban, which is considered a selective inhibitor of a single coagulation protein, may actually display nonhaemostatic anti-atherothrombotic effects;

(III) DOACs directly inhibiting Factor Xa or thrombin may be superior (in efficacy) to vitamin $\mathrm{K}$ antagonists (VKAs) in promoting adequate vascular protection.

\section{Factor Xa inbibition reduces PAR-mediated platelet activation}

A remarkable feature of the haemostatic system is the degree of redundancy and interdependence of the activation and regulatory processes involved. In addition to the multiple mechanisms for the coagulation to become activated, there 


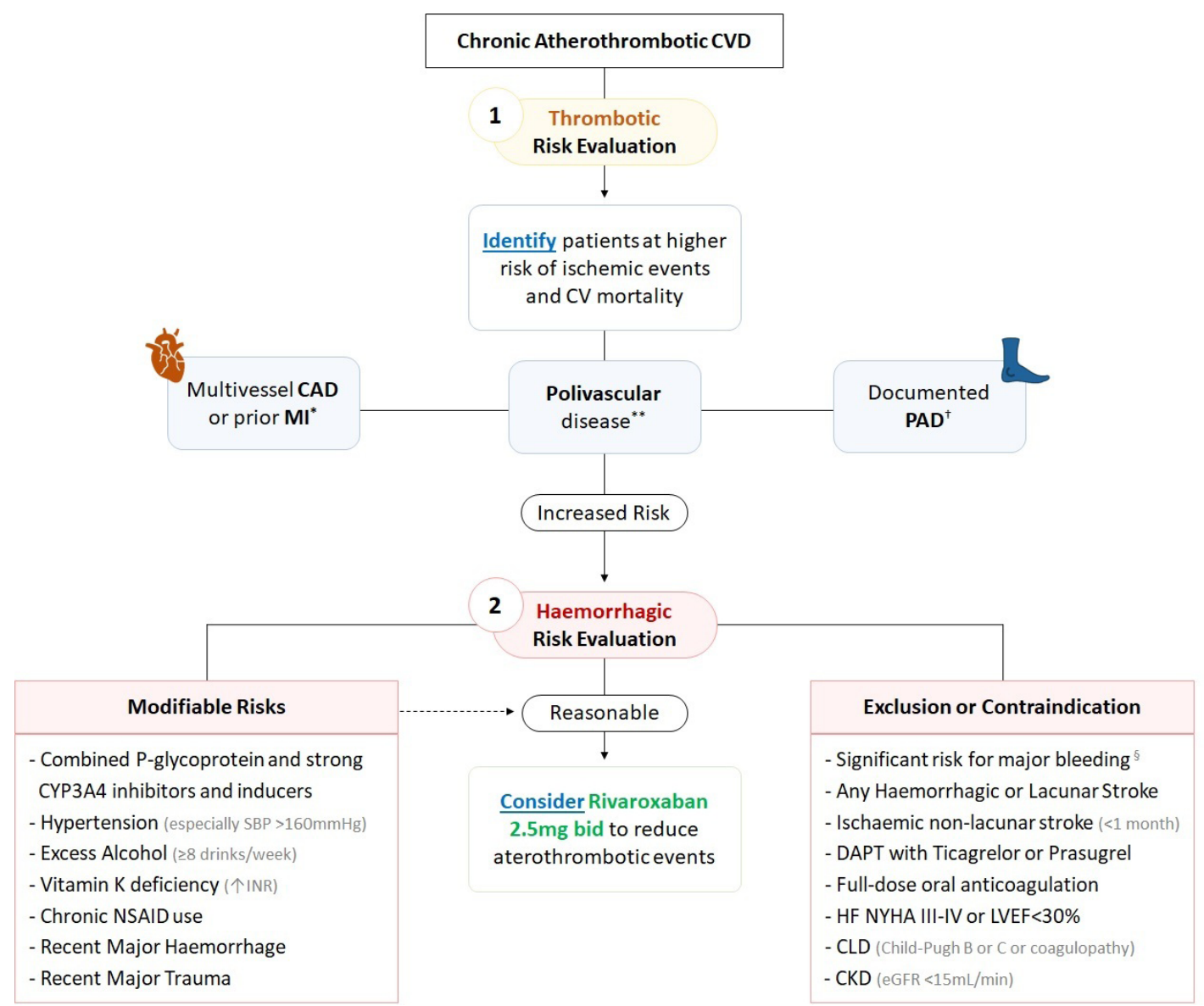

Figure 2 Algorithmic approach to identify potential candidates who may benefit from low-dose rivaroxaban. This algorithm was built based on the COMPASS trial inclusion and exclusion criteria, post-hoc analysis and current ESC recommendations. HF with reduced ejection fraction was not included as an isolated thrombotic risk criterion per se given the neutral results of the COMMANDER-HF trial (30). *, CAD in COMPASS was defined as prior MI or multivessel CAD with symptoms or with history of stable or unstable angina or multivessel percutaneous coronary intervention or multivessel CABG. ESC guidelines recommend DPI should be considered (IIa) when diffuse multivessel is present with other high-risk factors, namely either DM or CKD, and may be considered (IIb) when HF is present. Patients with a risk factor in addition to CAD, as previously defined, may have substantially higher residual risk and should or may be given DPI. However, it should be noted that COMPASS included patients with at least two additional risk factors, either DM, CKD or HF, when aged $<65$ years of age. **, Two or more of the following: CAD, PAD or stroke. ${ }^{\dagger}$, Documented by one of the following (I) history of symptomatic PAD (intermittent claudication) with either an ankle-brachial index $<0.90$ or $\geq 50 \%$ stenosis of peripheral artery by angiography or duplex ultrasound; (II) previous carotid revascularization (e.g., endarterectomy or stent) or asymptomatic carotid stenosis $\geq 50 \%$ documented by either angiography or duplex ultrasound; (III) previous aorto-femoral bypass surgery, limb bypass surgery or percutaneous transluminal angioplasty revascularization of the iliac or infrainguinal arteries; or (IV) previous limb or foot amputation for arterial vascular disease. $\$$, Active clinically significant bleeding; lesion or condition considered to be a significant risk for major bleeding (e.g., current or recent gastrointestinal ulceration, oesophageal varices, malignant neoplasm at high risk of bleeding, recent central nervous system or ophthalmic surgery, central nervous system arteriovenous malformations, aneurysms or major abnormalities and recent intracranial haemorrhage; congenital or acquired bleeding disorders; uncontrolled severe arterial hypertension; vascular retinopathy; bronchiectasis or history of pulmonary bleeding); rivaroxaban $2.5 \mathrm{mg}$ bid should be used with caution in patients aged $\geq 75 \mathrm{years}$ and/or weight $<60 \mathrm{~kg}$. ESC, European Society of Cardiology; HF, heart failure; CAD, coronary artery disease; MI, myocardial infarction; CABG, coronary artery bypass grafting; DPI, dual pathway inhibition; DM, diabetes mellitus; CKD, chronic kidney disease; PAD, peripheral artery disease; CVD, cardiovascular disease; CV, cardiovascular; SBP, systolic blood pressure; INR, international normalized ratio; NSAID, non-steroidal antiinflammatory drugs; DAPT, dual anti-platelet therapy; NYHA, New York Heart Association; LVEF, left ventricular ejection fraction; CLD, chronic liver disease; eGFR, estimated glomerular filtration rate. 


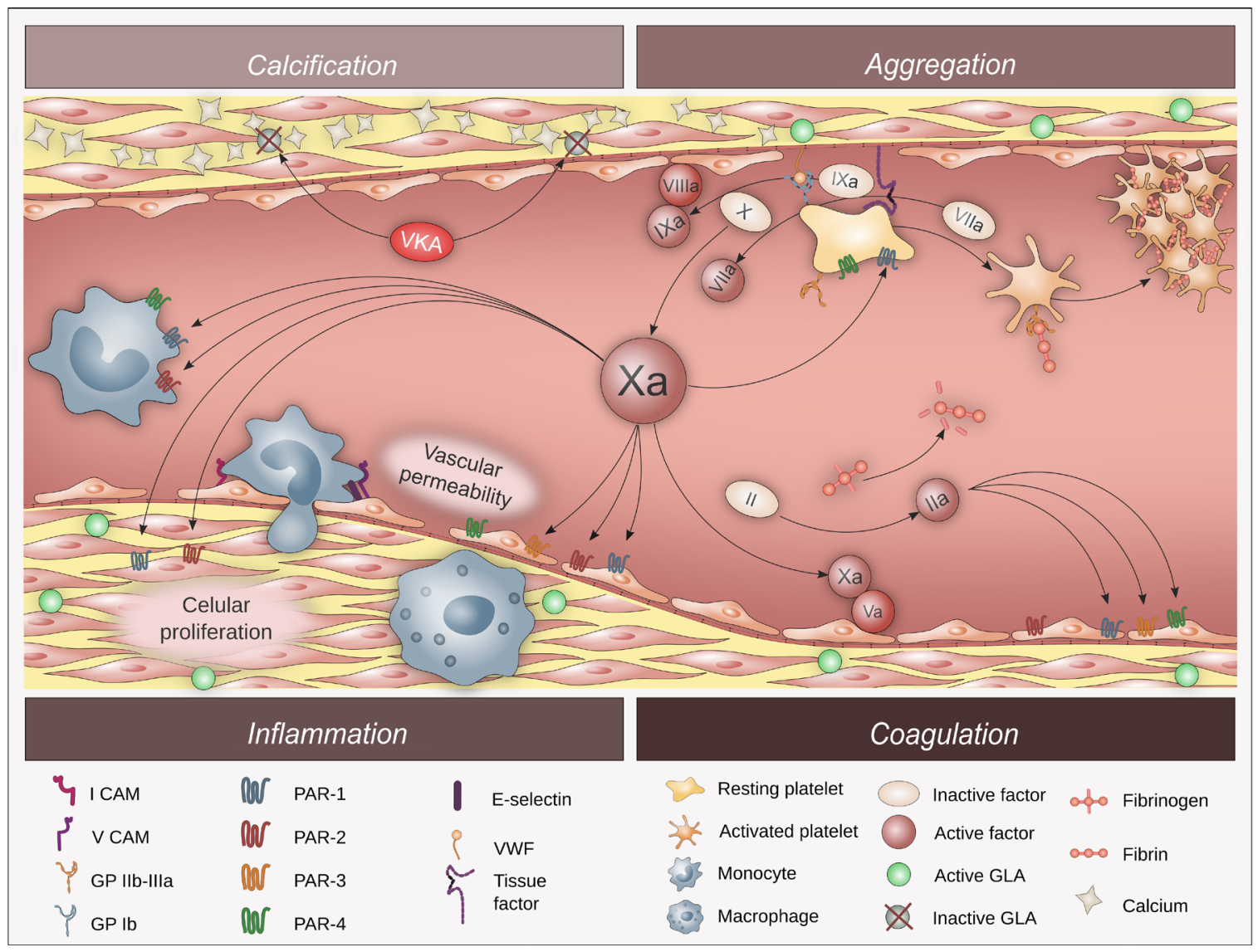

Figure 3 Mechanistic intertwining between coagulation cascade and platelets, focusing on the role of Factor Xa and PARs. PAR, proteaseactivated receptor; GLA, carboxyglutamic acid; ICAM, intracellular adhesion molecule; VCAM, vascular cell adhesion molecule; VKA, vitamin K antagonist; vW, von Willebrand factor; Gp, glycoprotein.

are also numerous and bidirectional associations between the coagulation system and platelets (32).

On the one hand, the coagulation system supports platelet activation and function in several ways. Thrombin, fibrinogen and von Willebrand factor (VWF) are coagulation proteins with a role in platelet activation or aggregation: (I) thrombin (Factor IIa) binds to PARs on the platelet surface and is the most potent endogenous platelet activator; (II) fibrinogen mediates glycoprotein IIb/IIIa platelet aggregation; and, (III) VWF, a protein produced constitutively as ultra-large VWF in endothelium (Weibel-Palade bodies), supports both platelet adhesion and aggregation. Anticoagulant drugs that reduce thrombin generation or inhibit thrombin can therefore be expected to reduce platelet activity.

On the other hand, activated platelets also have a vital procoagulant role (33-36): (I) during activation, platelet membrane phospholipids undergo rearrangement, exposing phosphatidylserine on the outer table of the membrane, thus providing a binding site for tissue factor and Factor VII, that further facilitate proteolysis and Factor $\mathrm{X}$ and prothrombin activation; (II) the platelet surface glycoprotein Ib-IX-V complex may promote Factor XI and XII activation; (III) platelet alpha-granules contain procoagulant factors (e.g., fibrinogen, VWF, Factor V).

\section{Factor Xa inbibition reduces PAR-mediated inflammation}

Thrombin and Factor Xa are serine proteases and, as such, enzymes that activate PARs by cleavage of their extracellular domain. PARs are highly expressed at the membrane surface of various cells involved in inflammation (37). These receptors, firstly described in 1991 as a thrombin receptor, are a subfamily of $G$ protein-coupled receptors 
that are activated by serine-dependent protease cleavage of their amino terminal sequence, which exposes the new $\mathrm{N}$-terminal sequence. The latter functions as a tethered ligand for different proteins. Once PARs are activated, an intracellular signal takes action, inducing changes in cell morphology, adhesion, secretion, growth, proliferation and/or motility (38).

There are four known mammalian members of the unique PAR family, predominantly expressed in platelets, leukocytes, endothelial and vascular smooth muscle cells, as well as in myocytes, cardiac fibroblasts, and neurons. Protease signalling through PARs contributes to both homeostasis and disease states. Indeed, activation of PARs is important for normal haemostasis and vascular tone, but has also been implicated in pathological thrombosis, atherosclerosis, and cardiac hypertrophy and remodelling (39-41). Thrombin activates PAR-1, PAR-3 and PAR-4, while Factor Xa activates PAR-1 and PAR-2. The potential atherothrombotic effects of Factor $\mathrm{Xa}$, subsequent to the activation of the coagulation cascade and PARs, are presented in Figure 3.

The activation of the PARs on endothelial cells induces the secretion of cytokines such as IL-1 and IL6 , and increases the expression of cell adhesion molecules, particularly vascular cell adhesion molecule (VCAM)-1, intracellular adhesion molecule (ICAM)-1, and E-selectin. These adhesion molecules enhance vascular endothelium barrier permeability and facilitate the interaction between endothelial cells and circulating leukocytes, promoting monocyte adhesion and migration from the bloodstream into the subendothelial space of the arterial walls. The activation of PARs on vascular smooth muscle cells promotes deleterious and atherogenic signalling responses such as cell growth and proliferation, and accumulation of dysfunctional extracellular matrix. PAR signalling in monocytes and macrophages promotes the activation of proliferative and inflammatory pathways. Altogether, the evidence supports that PAR-mediated signalling has a key role in atherosclerosis progression and atherothrombotic events. Interestingly, a study in an animal model (apolipoprotein E-deficient mice) has suggested that the pharmacological inhibition of Factor Xa with rivaroxaban led to regression of advanced atherosclerosis and enhanced plaque stability (42).

\section{Factor Xa inbibition promotes vascular protection}

Vitamin K (VK) dependent proteins are Factors II, VII, IX,
$\mathrm{X}$, and proteins $\mathrm{C}$ and $\mathrm{S}$. One could expect that reducing the activation of Factor $\mathrm{X}$ and thrombin through VKA would share the above-mentioned pleiotropic effects observed with selective Factor inhibition with DOACs. However, the anti-atherogenic potential of VKA compared to DOACs may be limited for two reasons: (I) VKA have been shown to promote vascular calcification (43); and (II) VKA do not seem to alter the binding capacity of VK dependent factors to PARs (40). These limitations are further discussed below.

During the atherosclerotic process, vascular smooth muscle cells undergo a phenotypic conversion to proliferative synthetic cells resembling active osteoblasts, thus generating extracellular matrix and promoting vascular calcification. Proteins known to inhibit this process include matrix Gla protein, Gla-rich protein and osteocalcin. These proteins require gamma-glutamyl carboxylation of glutamic acid residues to become functionally active. In turn, this carboxylation process requires VK as its cofactor. As VKA block this reaction, these anticoagulants may facilitate vascular calcification, notably in blood vessels damaged by atherosclerosis (43). Indeed, this is the rationale for trials testing VK supplementation $(44,45)$. Furthermore, two trials of a Factor Xa inhibitor versus warfarin have shown that the latter is associated with greater progression of total plaque volume and calcified plaque volume in coronary arteries $(46,47)$.

The activity of VK-dependent coagulation proteins depends on their capacity to bind to negatively charged cell membranes such as activated platelets. Binding to these membranes is enhanced if certain glutamic acid residues of these proteins are modified by gamma-glutamyl carboxylase, creating a gamma carboxyglutamic acid-rich (Gla) domain. VK is a cofactor for this carboxylase. In the absence of VK (or if VKA are being taken), the VKdependent coagulation proteins will lack the Gla-rich domain and, therefore, convert only minimal quantities of preprothrombin to prothrombin, resulting in an anticoagulant effect. While Gla domain-less thrombin and Factor $\mathrm{Xa}$ have diminished coagulation activity, the Gla-rich domain is not required for the serine protease activity that activates the PARs. For this reason, VKA do not compromise the atherothrombotic phenomena that result from the activation of PARs in endothelial cells, vascular smooth muscle cells, and inflammatory cells inside the arterial wall. Treatment with a direct thrombin inhibitor will cause both attenuated coagulation and cellular signalling through PAR1 with unaltered signalling 
capacity for Factor Xa. In contrast, since direct Factor Xa inhibition alters both coagulation and PAR1 and PAR2 signalling, greater anti-atherothrombotic effects may be expected from this intervention (40).

\section{Conclusions}

Rivaroxaban, a DOAC acting by exclusively inhibiting Factor $\mathrm{Xa}$, was developed to prevent thrombus formation. However, two landmark trials $(11,23)$ have shown that rivaroxaban $2.5 \mathrm{mg}$ twice daily (the so-called vascular protection dose) in secondary prevention led to a significant improvement in $\mathrm{CV}$ outcomes in addition to antiplatelet therapy (48). These findings support an antiatherothrombotic effect considering the interactions between coagulation and inflammation that are mediated by PAR (4,39-41).

Therefore, it may be more appropriate to consider rivaroxaban as an agent that exclusively inhibits Factor Xa, a serine protease with ancillary effects beyond thrombin formation, namely PAR-mediated activation of atherogenicpromoting key players (platelets, endothelial cells, vascular smooth muscle cells, and macrophages) (4,39-41).

\section{Acknowledgments}

Funding: None.

\section{Footnote}

Reporting Checklist: The authors have completed the Narrative Review reporting checklist. Available at http:// dx.doi.org/10.21037/cdt-20-859

Peer Review File: Available at http://dx.doi.org/10.21037/cdt20-859

Conflicts of Interest: All authors have completed the ICMJE uniform disclosure form (available at http://dx.doi. org/10.21037/cdt-20-859). CMTA has received honoraria for consultancy and/or speaker fees from Amgen, AstraZeneca, Bayer, Boehringer-Ingelheim, BMS-Pfizer, and Daiichi-Sankyo. CMTA serves as an unpaid editorial board member of Cardiovascular Diagnosis and Therapy from Jul 2019 to Jun 2021. The other authors have no conflicts of interest to declare.

Ethical Statement: The authors are accountable for all aspects of the work in ensuring that questions related to the accuracy or integrity of any part of the work are appropriately investigated and resolved.

Open Access Statement: This is an Open Access article distributed in accordance with the Creative Commons Attribution-NonCommercial-NoDerivs 4.0 International License (CC BY-NC-ND 4.0), which permits the noncommercial replication and distribution of the article with the strict proviso that no changes or edits are made and the original work is properly cited (including links to both the formal publication through the relevant DOI and the license). See: https://creativecommons.org/licenses/by-nc-nd/4.0/.

\section{References}

1. Alberts MJ, Bhatt DL, Mas JL, et al. Three-year followup and event rates in the international REduction of Atherothrombosis for Continued Health Registry. Eur Heart J 2009;30:2318-26.

2. Steg PG, Bhatt DL, Wilson PW, et al. Oneyear cardiovascular event rates in outpatients with atherothrombosis. JAMA 2007;297:1197-206.

3. van Kuijk JP, Flu WJ, Welten GM, et al. Long-term prognosis of patients with peripheral arterial disease with or without polyvascular atherosclerotic disease. Eur Heart J 2010;31:992-9.

4. Olie RH, van der Meijden PEJ, Ten Cate H. The coagulation system in atherothrombosis: implications for new therapeutic strategies. Res Pract Thromb Haemost 2018;2:188-98.

5. Kaasenbrood L, Boekholdt SM, van der Graaf Y, et al. Distribution of estimated 10-year risk of recurrent vascular events and residual risk in a secondary prevention population. Circulation 2016;134:1419-29.

6. Kaasenbrood L, Bhatt DL, Dorresteijn JAN, et al. Estimated life expectancy without recurrent cardiovascular events in patients with vascular disease: the SMARTREACH model. J Am Heart Assoc 2018;7:e009217.

7. Bansilal S, Bonaca MP, Cornel JH, et al. Ticagrelor for secondary prevention of atherothrombotic events in patients with multivessel coronary disease. J Am Coll Cardiol 2018;71:489-96.

8. Sabatine MS, Giugliano RP, Keech AC, et al. Evolocumab and clinical outcomes in patients with cardiovascular disease. N Engl J Med 2017;376:1713-22.

9. Ridker PM, Everett BM, Thuren T, et al. Antiinflammatory therapy with canakinumab for 
atherosclerotic disease. N Engl J Med 2017;377:1119-31.

10. Zinman B, Wanner C, Lachin JM, et al. Empagliflozin, cardiovascular outcomes, and mortality in type 2 diabetes. N Engl J Med 2015;373:2117-28.

11. Eikelboom JW, Connolly SJ, Bosch J, et al. Rivaroxaban with or without aspirin in stable cardiovascular disease. $\mathrm{N}$ Engl J Med 2017;377:1319-30.

12. Tardif JC, Kouz S, Waters DD, et al. Efficacy and safety of low-dose colchicine after myocardial infarction. $\mathrm{N}$ Engl J Med 2019;381:2497-505.

13. Marso SP, Baeres FMM, Bain SC, et al. Effects of liraglutide on cardiovascular outcomes in patients with diabetes with or without heart failure. J Am Coll Cardiol 2020;75:1128-41.

14. Gibson CM, Chakrabarti AK, Mega J, et al. Reduction of stent thrombosis in patients with acute coronary syndromes treated with rivaroxaban in ATLAS-ACS 2 TIMI 51. J Am Coll Cardiol 2013;62:286-90.

15. Savarese G, De Ferrari GM, Rosano GM, et al. Safety and efficacy of ezetimibe: A meta-analysis. Int J Cardiol 2015;201:247-52.

16. Anand SS, Eikelboom JW, Dyal L, et al. Rivaroxaban plus aspirin versus aspirin in relation to vascular risk in the COMPASS trial. J Am Coll Cardiol 2019;73:3271-80.

17. Smith P, Arnesen H, Holme I. The effect of warfarin on mortality and reinfarction after myocardial infarction. $\mathrm{N}$ Engl J Med 1990;323:147-52.

18. Hurlen M, Abdelnoor M, Smith P, et al. Warfarin, aspirin, or both after myocardial infarction. N Engl J Med 2002;347:969-74.

19. Wallentin L, Wilcox RG, Weaver WD, et al. Oral ximelagatran for secondary prophylaxis after myocardial infarction: the ESTEEM randomised controlled trial. Lancet 2003;362:789-97.

20. Oldgren J, Budaj A, Granger CB, et al. Dabigatran vs. placebo in patients with acute coronary syndromes on dual antiplatelet therapy: a randomized, double-blind, phase II trial. Eur Heart J 2011;32:2781-9.

21. Alexander JH, Lopes RD, James S, et al. Apixaban with antiplatelet therapy after acute coronary syndrome. N Engl J Med 2011;365:699-708.

22. Sherwood MW, Lopes RD, Sun JL, et al. Apixaban following acute coronary syndromes in patients with prior stroke: insights from the APPRAISE-2 trial. Am Heart J. 2018;197:1-8.

23. Mega JL, Braunwald E, Wiviott SD, et al. Rivaroxaban in patients with a recent acute coronary syndrome. $\mathrm{N}$ Engl J Med 2012;366:9-19.
24. Bonaca MP, Bauersachs RM, Anand SS, et al. Rivaroxaban in peripheral artery disease after revascularization. N Engl J Med 2020;382:1994-2004.

25. Mega JL, Braunwald E, Mohanavelu S, et al. Rivaroxaban versus placebo in patients with acute coronary syndromes (ATLAS ACS-TIMI 46): a randomised, double-blind, phase II trial. Lancet 2009;374:29-38.

26. Leon MB, Baim DS, Popma JJ, et al. A clinical trial comparing three antithrombotic-drug regimens after coronary-artery stenting. Stent Anticoagulation Restenosis Study Investigators. N Engl J Med 1998;339:1665-71.

27. Hiatt WR, Fowkes FG, Heizer G, et al. Ticagrelor versus clopidogrel in symptomatic peripheral artery disease. $\mathrm{N}$ Engl J Med 2017;376:32-40.

28. Bonaca MP, Scirica BM, Creager MA, et al. Vorapaxar in patients with peripheral artery disease: results from TRA2\{degrees\}P-TIMI 50. Circulation 2013;127:1522-9, 1529.e1-6.

29. Anand SS, Bosch J, Eikelboom JW, et al. Rivaroxaban with or without aspirin in patients with stable peripheral or carotid artery disease: an international, randomised, double-blind, placebo-controlled trial. Lancet 2018;391:219-29.

30. Zannad F, Anker SD, Byra WM, et al. Rivaroxaban in patients with heart failure, sinus rhythm, and coronary disease. N Engl J Med 2018;379:1332-42.

31. Esmon CT. Targeting factor $\mathrm{Xa}$ and thrombin: impact on coagulation and beyond. Thromb Haemost 2014;111:625-33.

32. Badimon L, Padró T, Vilahur G. Atherosclerosis, platelets and thrombosis in acute ischaemic heart disease. Eur Heart J Acute Cardiovasc Care 2012;1:60-74.

33. Suehiro K, Smith JW, Plow EF. The ligand recognition specificity of beta3 integrins. J Biol Chem 1996;271:10365-71.

34. Lundblad RL, White GC. The interaction of thrombin with blood platelets. Platelets 2005;16:373-85.

35. Reininger AJ, Heijnen HF, Schumann H, et al. Mechanism of platelet adhesion to von Willebrand factor and microparticle formation under high shear stress. Blood 2006;107:3537-45.

36. Kottke-Marchant K, Corcoran G. The laboratory diagnosis of platelet disorders. Arch Pathol Lab Med 2002;126:133-46.

37. Krishnaswamy S. The transition of prothrombin to thrombin. J Thromb Haemost 2013;11 Suppl 1:265-76.

38. Soh UJ, Dores MR, Chen B, et al. Signal transduction by protease-activated receptors. Br J Pharmacol 
2010;160:191-203.

39. Leger AJ, Covic L, Kuliopulos A. Protease-activated receptors in cardiovascular diseases. Circulation 2006;114:1070-7.

40. Spronk HM, de Jong AM, Crijns HJ, et al. Pleiotropic effects of factor Xa and thrombin: what to expect from novel anticoagulants. Cardiovasc Res 2014;101:344-51.

41. Heuberger DM, Schuepbach RA. Protease-activated receptors (PARs): mechanisms of action and potential therapeutic modulators in PAR-driven inflammatory diseases. Thromb J 2019;17:4.

42. Posthuma JJ, Posma JJN, van Oerle R, et al. Targeting coagulation factor $\mathrm{Xa}$ promotes regression of advanced atherosclerosis in apolipoprotein-E deficient mice. Sci Rep 2019;9:3909.

43. Andrews J, Psaltis PJ, Bayturan O, et al. Warfarin use is associated with progressive coronary arterial calcification: insights from serial intravascular ultrasound. JACC Cardiovasc Imaging 2018;11:1315-23.

Cite this article as: Rocha BML, da Cunha GJL, Aguiar CMT. A narrative review of low-dose rivaroxaban in patients with atherothrombotic cardiovascular disease: vascular protection beyond anticoagulation. Cardiovasc Diagn Ther 2021;11(1):130-141. doi: 10.21037/cdt-20-859
44. Lees JS, Chapman FA, Witham MD, et al. Vitamin K status, supplementation and vascular disease: a systematic review and meta-analysis. Heart 2019;105:938-45.

45. Roumeliotis S, Dounousi E, Eleftheriadis T, et al. Association of the inactive circulating matrix gla protein with vitamin $\mathrm{K}$ intake, calcification, mortality, and cardiovascular disease: A Review. Int J Mol Sci 2019;20:628.

46. Lee J, Nakanishi R, Li D, et al. Randomized trial of rivaroxaban versus warfarin in the evaluation of progression of coronary atherosclerosis. Am Heart J 2018;206:127-30.

47. Win TT, Nakanishi R, Osawa K, et al. Apixaban versus warfarin in evaluation of progression of atherosclerotic and calcified plaques (prospective randomized trial). Am Heart J 2019;212:129-33.

48. Barrios V, Almendro-Delia M, Facila L, et al. Rivaroxaban: searching the integral vascular protection. Expert Rev Clin Pharmacol 2018;11:719-28. 